\title{
APPLICATION OF THE GALERKIN-VLASOV METHOD TO THE FLEXURAL ANALYSIS OF SIMPLY SUPPORTED RECTANGULAR KIRCHHOFF PLATES UNDER UNIFORM LOADS
}

\author{
N. N. Osadebe ${ }^{1}$, C. C. Ike ${ }^{2,},{ }^{*}$ H. Onah ${ }^{3}$, C. U. Nwoji4 and F. O. Okafor ${ }^{5}$

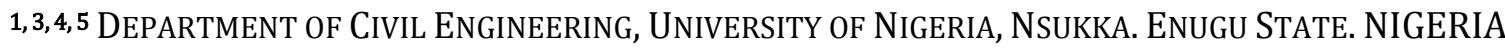 \\ 2, Dept of Civil EngineERing, Enugu State University of SCIENCE \& TeChNology, EnUgu,. EnUgu State. NigERIA \\ E-mail addresses:11mkemamaka@yahoo.co.uk, ${ }^{2}$ ikecc2007@yahoo.com,3hyginus.onah@unn.edu.ng, \\ ${ }_{4}$ clifford.nwoji@unn.edu.ng,5 fidelis.okafor@unn.edu.ng
}

\begin{abstract}
Plates are important structural elements used to model bridge decks, retaining walls, floor slabs, spacecraft panels, aerospace structures, and ship hulls amongst. Plates have been modelled using three dimensional elasticity theory, Reissner's theory, Kirchhoff theory, Shimpi's theory, Von Karman's theory, etc. The resulting plate equations have also been solved using classical and numerical techniques.In this research, the Galerkin-Vlasov variational method was used to present a general formulation of the Kirchhoff plate problem with simply supported edges and under distributed loads. The problem was then solved to obtain the displacements, and the bending moments in a Kirchhoff plate with simply supported edges and under uniform load. Maximum values of the displacement and the bending moments were found to occur at the plate center. The Galerkin Vlasov solutions for a rectangular simply supported Kirchhoff plate carrying uniform load was found to be exactly identical with the Navier double trigonometric series solution.
\end{abstract}

Keywords: Kirchhoff Plate, Reissner Plate, Galerkin-Vlasov method, Navier double trigonometric series method, uniform load.

\section{INTRODUCTION}

Plates are initially flat structural members bounded by two parallel planes, called faces and an edge[1]. Plates have been considered as two dimensional extensions of beams. This two dimensional structural action of plates results in lighter structures, and this offers immense benefits. Plates can be submitted to static loads, dynamic or transient (time dependent) loads or in plane loads, resulting in three types of analysis namely - static or elasticity analysis, dynamic analysis and stability analysis.

The focus of this paper is the static elasticity analysis of plates since the loads are considered to be static. The aims and objectives of the elasticity analysis of plates are:

(i) the determination of deflection due to applied static loads and the critical values of the deflection (ii) the determination of the internal force functions such as the bending moments, and their critical values.

Plates have been classified based on their shapes as rectangular, triangular, circular, skew, square, elliptical etc plates, and based on their material properties as isotropic, anisotropic or orthotropic, homogeneous, heterogeneous, etc. Plates have also been classified based in their thicknesses as thin plates, moderately thick plates and thick plates [2-4]. Plates are used in bridge decks, retaining walls, ship decks, hulls, aircraft and spacecraft panels, and are thus important structural forms in civil, mechanical, aerospace, and naval engineering.The focus of this paper shall be on rectangular thin plates made of homogeneous, isotropic material.

\subsection{Justification of the Galerkin-Vlasov's Method}

The Galerkin-Vlasov method is proposed in this work due to the merits of the method which include: 
(i) Due to the relative ease in selecting shape functions for almost all boundary conditions encountered in practice, Galerkin-Vlasov method can be considered more universal than the other variational methods like Ritz Variational Method, Galerkin Variational Method and the Kantorovich Variational method [5]

(ii) Further simplification results in numerical computation in the method due to the quasiorthogonality of the eigen functions of the vibrating beam used as the basis function in the method [5].

(iii) The problem is reduced to the evaluation of the integrals of simple functions with quasiorthogonal properties.

(iv) The choice of appropriate basis functions simply reduces to a matter of choosing beam functions with identical boundary conditions as those of the plate.

(v) The accuracy of the method is excellent, despite the relative ease of the numerical computation involved [5].

(vi) The method has considerable merits in the manual solutions of plate flexure problems [5].

However, the method has the disadvantage that as a variational method, it is not suited for automated computer applications [5].

\subsection{Research Problem}

The research problem considered in this paper is stated as follows:

Given a rectangular thin plate made of homogeneous, isotropic material, we wish to apply the GalerkinVlasov variational technique to solve the elasticity problem of finding the deflection and the internal bending moments, and their critical values for simply supported edges and uniformly distributed static loads over the entire surface of the plate.

Mathematically, the research problem is to present a Galerkin-Vlasov variational formulation of the Kirchhoff plate problem given by Equation (1)for rectangular plates with simply supported edges.

$$
D \nabla^{2} \nabla^{2} w(x, y)=P_{z}(x, y)
$$

where $w(x, y)$ is the transverse displacement of the plate middle surface,

$P_{z}(x, y)$ is the transverse load distribution on the plate,

$D$ is the flexural rigidity of the plate and $\nabla^{2}$ is the Laplacian operator.

$\nabla^{2} \nabla^{2}$ is the biharmonic operator.

\subsection{Research Aims and Objectives}

The aims and objectives of this study include:

(i) to propose a Galerkin-Vlasov variational formulation of the Kirchhoff plate problem for static loads.

(ii) to solve the Galerkin-Vlasov variational problem and find the deflection function for Kirchhoff plates with simply supported edges and under uniform loads.

(iii) to use the moment - displacement relations for Kirchhoff plates to find the bending moment functions, and their critical values.

(iv) to compare the results of the Galerkin-Vlasov solution with the results from literature sources.

\section{LITERATURE REVIEW}

Several types of plate theories exist. The basic idea of all plate theories is to simplify the three dimensional problem of plates described by the theory of elasticity to two dimensional approximations; usually done by integrating out a dimension.

Accordingly, a review of existing literature shows the following plate theories: thin plate theories, moderately thick plate theories and thick plate theories [1, 2, 3]. We present a review of the following plate theories: Exact plate theory, Reissner's sixth order plate theory, Von Karman plate theory, Mindlin plate theory, Shimpi's refined plate theory and Kirchhoff plate theory.

\subsection{Exact Theory of Plates}

This is defined by the three dimensional elasticity equation of plates. The exact theory of plates is a three dimensional presentation of the plate flexure problem based on a simultaneous satisfaction of the diffferential equations of equilibrium, the kinematic equations and the material constitutive laws [5],[1]. Th.e governing equations are a system of fifteen differential equations, which need to be solved subject to the conditions of restraint and the loading. Three approaches are used to solve the resulting equations, namely - stress based approach, displacement based approach and mixed approach. The resulting governing equations are complicated and difficult to solve in analytical form [1], [5].

\subsection{Reissner's Sixth Order Plate Theory}

Reissner's stress based theory of plate presents an integration prblem of sixth order. Reissner's plate theory for isotropic plates is a system of three simultaneous differential equations. [5] [6].In the 
Reissner's plate theory, the distributions of stress components through the plate thickness are assumed to be:

$$
\begin{gathered}
\sigma_{x x}=z \cdot \frac{12 \mu_{x x}}{h^{3}}, \sigma_{y y}=z \cdot \frac{12 \mu_{y y}}{h^{3}}, \tau_{x y}=z \cdot \frac{12 \mu_{x y}}{h^{3}} \\
\tau_{x z}=\frac{3 Q_{x}}{2 h}\left(1-\left(\frac{2 z}{h}\right)^{2}\right), \tau_{y z}=\frac{3 Q_{x}}{2 h}\left(1-\left(\frac{2 z}{h}\right)^{2}\right), \\
\sigma_{z z}=\frac{-q}{4}\left[2-3\left(\frac{2 z}{h}\right)+\left(\frac{2 z}{h}\right)^{3}\right]
\end{gathered}
$$

In (2), $\sigma_{x x}, \sigma_{y y}, \sigma_{z z}$ are the normal stresses and $\tau_{x y}, \tau_{y z}$ $\tau_{x z}$ are the shear stresses, $h$ is the plate thickness, $M_{X x}$ $M_{y y}$ are the bending moment distributions, $Q_{x}, Q_{y}$ are shear forces distributions and $z$ is the position of an arbitrary point on the plate thickness.

Thus, the assumed distribution of in plane stresses are linear, the transverse shear stresses are quadratic and the transverse normal stress is cubic in the plate thickness direction. These stress components satisfy the stress equilibrium equations of three dimensional elasticity theory. The transverse displacement of Reissner's theory is a function of the three space coordinates of the plate, complicating its determination. In order to simplify the governing equations, Reissner introduced the thickness integrated transvese displacement $w(x, y)$ as:

$$
w(x, z)=\frac{3}{2 h} \int_{-h / 2}^{h / 2} w(x, y, z)\left(1-\left(\frac{2 h}{h}\right)^{2}\right) d z
$$

Reissner applied the Castigliano's theorem of least work to the energy functional of the plate to obtain the following three simultaenous differential equations [5]:

$$
\begin{aligned}
& D \nabla^{2} \nabla^{2} w=p_{z}-\frac{h^{2}}{10}\left(\frac{2-\mu}{1-\mu}\right) \nabla^{2} p_{z} \\
& q_{x}=-D \frac{\partial \nabla^{2}}{\partial x} w+\frac{h^{2} \nabla^{2}}{10} q_{x}-\frac{h^{2}}{10}\left(\frac{1}{1-\mu}\right) \frac{\partial p_{z}}{\partial x} \\
& q_{y}=-D \frac{\partial \nabla^{2}}{\partial y} w+\frac{h^{2} \nabla^{2}}{10} q_{y}-\frac{h^{2}}{10}\left(\frac{1}{1-\mu}\right) \frac{\partial p_{z}}{\partial y}
\end{aligned}
$$

The bending and twisting moments are given by

$$
\begin{gathered}
M_{x x}=-D\left(\frac{\partial^{2} w}{\partial x^{2}}+\mu \frac{\partial^{2} w}{\partial y^{2}}\right)+\frac{h^{2}}{5} \frac{\partial q_{x}}{\partial x} \\
-\frac{h^{2}}{10}\left(\frac{h^{2}}{1-\mu}\right) p_{z} \\
M_{y y}=-D\left(\frac{\partial^{2} w}{\partial x^{2}}+\mu \frac{\partial^{2} w}{\partial y^{2}}\right)+\frac{h^{2}}{5} \frac{\partial q_{y}}{\partial y} \\
-\frac{h^{2}}{10}\left(\frac{\mu}{1-\mu}\right) p_{z} \\
M_{x y}=-D(1-\mu) \frac{\partial^{2} w}{\partial x \partial y}+\frac{h^{2}}{10}\left(\frac{\partial q_{y}}{\partial x}+\frac{\partial q_{y}}{\partial y}\right)
\end{gathered}
$$

The Reissner's plate theory is an extension of the Kirchhoff - Love plate theory, accounting for first order shear effects. Reissner plate theory gives stress and strain fields that are consistent with elasticity solutions; and are applicable to moderately thick plates. However, the governing equations involve complicated systems of partial differential equations for which satisfactory solutions are not found for complex loading and restraint conditions [5], [1], [2].

\subsection{Von Karman Plate Theory}

The Von Karman large deflection plate theory yields the system of equilibrium and compatiblity equation given by

$$
\begin{aligned}
& \frac{D}{h} \nabla^{4} w=L(w, \phi)+\frac{p_{z}}{h} \\
& \frac{1}{E} \nabla^{2} \phi(x, y)=-\frac{1}{2} L(w, w)
\end{aligned}
$$

where $L$ is a differential generator defined as

$$
L(w, \phi)=\frac{\partial^{2} w}{\partial x^{2}} \frac{\partial^{2} \phi}{\partial x^{2}}+\frac{\partial^{2} w}{\partial y^{2}} \frac{\partial^{2} \phi}{\partial y^{2}}-2 \frac{\partial^{2} w}{\partial x \partial y} \frac{\partial^{2} \phi}{\partial x \partial y}
$$

In (12), $w(x, y)$ is the transverse displacement of the plate, $p_{z}$ is the transverse load distribution, $D$ is the flexural rigidity of the plate, $E$ is the modulus of elasticity and $\phi$ is the Airy's stress function.

Von Karman plate equations are coupled nonlinear partial differential equations of the fourth order.Von Karman's large deflection plate theory considers the longitudinal displacement of the middle plane caused by the deflection and hence the middle plane stress and strain. The Von Karman's equations cannot; in general be solved directly in analytical form, except using some approximate methods $[3,5]$.

\subsection{Mindlin Plate Theory}

The Mindlin plate theory, which is a first order shear deformable theory, does not require that the crosssection remains orthogonal to the middle surface which is the neutral surface after deformation [7]. Therefore, $\varepsilon_{x z} \neq 0$ and $\varepsilon_{y z} \neq 0$. The theory assumes that the state of deformation is described by the transverse displacements in the $z$ direction of the middle surface $w(x, y)$ at $\mathrm{z}=0$ and the notations $\theta_{x}$ and $\theta_{y}$ of the middle surface [8]. The displacement field at a generic point of the plate are $w=$ $w(x, y, z=0)=w(x, y), u(x, y, z)=z \theta_{x}(x, y)$ and $v=$ $z \theta_{y}(x, y)$ and where $u, v$ and $w$ are the displacement $[8,9]$ components in the $x, y$ and $z$ directions respectively.

The governing equations of isotropic Mindlin plaes are: 


$$
\begin{aligned}
\nabla^{2} w+\frac{\partial \theta_{x}}{\partial x}+\frac{\partial \theta_{y}}{\partial y} & =\frac{-q}{k G h} \\
\nabla^{2}\left(\frac{\partial \theta_{x}}{\partial x}+\frac{\partial \theta_{y}}{\partial y}\right) & =\frac{-q}{D} \\
D\left(\frac{\partial^{2} \theta_{x}}{\partial x^{2}}+\frac{\partial^{2} \theta_{y}}{\partial y}\right) & +\frac{D(1-\mu)}{2}\left(\frac{\partial^{2} \theta_{x}}{\partial x^{2}}+\frac{\partial^{2} \theta_{y}}{\partial y \partial x}\right) \\
= & k G h\left(\theta_{x}+\frac{\partial w}{\partial x}\right)
\end{aligned}
$$

The shear strain and shear stress variation across the plate thickness is considered in the Mindlin plate theory. However the assumption of constant shear strain variation over the plate thickness violates the predictions of structural theory since the shear stress is known to be parabolic over the plate thickness. This discrepancy is corrected using a shear correction factor $k$. Also the Mindlin plate theory contradicts the shear - free boundary condition on the plate surfaces. The governing partial differential equations are difficult to solve mathematically even for simple loads and restaint conditions.Mindlin plate theory yields a system of three differential equations in terms of three unknown displacement parameters for isotropic plates.

\subsection{Shimpi's Refined Plate Theory [Shimpi's (RPT)]}

Shimpi [10] developed a new refined plate theory and its two variants, variationally consistent variant and variational inconsistent variant. Shimpi's variationally consistent RPT equations are:

$$
\begin{aligned}
& \nabla^{2} \nabla^{2}=\frac{q}{D} \\
& \nabla^{2} w_{z}=\frac{-h^{2}}{5(1-\mu)} \frac{q}{D}
\end{aligned}
$$

where $w_{b}$ is the displacement due to bending, $w_{s}$ is the displacement due to shear, $q$ is the transverse load, $D$ is the flexural rigidity $\mu$ is the Poisson's ratio and $h$ is the plate thickness.

Shimpi's variationally inconsistent plate equations are:

$$
\begin{aligned}
& \nabla^{2} \nabla^{2} w_{b}=\frac{q}{D} \\
& w=w_{z}-\left(\frac{h^{2}}{5(1-\mu)}\right) \nabla^{2} w_{b}
\end{aligned}
$$

\subsection{Kirchhoff Plate Theory}

The Kirchhoff plate theory assumes as follows:

(i) the plate thickness is small, compared to the other dimensions, the normal stress is in the plane of the plate.

(ii) the deflection is smallcompared to the plate thickness. (iii) the middle surface is free from deformation.

(iv) normals to the middle surface remain normal to the middle surface during and after deformation.

The equilibrium equation for Kirchhoff plate is given by, the fourth order partial differential equation, Equation (1).Literature review shows that plates are analysed for elastic, dynamic and stability behaviour, using Navier's double trigonometric series method. Other methods used for the analysis of plates include:Levy Single Trigonometric Series, Finite Difference, Improved Finite Difference, Boundary Element, Finite Element, Finite Strip, Weighted Residual,Polynomial Splines, Differential Quadrature andEnergy Minimization.

\section{RESEARCH METHODOLOGY}

\subsection{Galerkin-Vlasov Method}

This research uses the Galerkin-Vlasov method to solve the Kirchhoff plate bending problem given by Equation (1).

In variational form,Equation (1) can be written as:

$$
\iint_{R}\left(D \nabla^{2} \nabla^{2} \mathrm{w}-\mathrm{p}\right) \delta w d x d y=0
$$

where $\delta w$ is a small virtual displacement

The Galerkin-Vlasov method assumes that the plate deflection and the load are expanded using the orthorgonal functions $X_{m(x)}$ and $Y_{n(y)}$ of a freely vibrating beam that is identical with the ends of the plate.

Thus,

$$
w(x, y)=\sum_{m}^{\infty} \sum_{n}^{\infty} w_{m n} X_{m}(x) Y_{n}(y) m, n
$$

where $W_{m n}$ are the unknown parameters of the displacement function.

$p(x, y)=\sum_{m}^{\infty} \sum_{n}^{\infty} p_{m n} X_{m}(x) Y_{n}(y)$

where $p_{m n}$ are the parameters of the load distribution. Then the variational Kirchhoff plate problem, using the Galerkin-Vlasov method becomes, in general,

$$
\begin{aligned}
& \iint\left[D\left(D \nabla^{2} \nabla^{2} \sum \sum w_{m n} X_{m}(x) Y_{n}(y)\right)\right. \\
& \left.-\sum \sum p_{m n} X_{m}(x) Y_{n}(y)\right] X_{i}(x) Y_{k} d x d y=0
\end{aligned}
$$

$W_{m n}$ and $p_{m n}$ are parameters of the displacement function $w(x, y)$ and the transverse load distribution $p(x, y)$ respectively, and since they don't depend upon the space variables, they could be factored out of the double integration in Equation (23) to obtain: 


$$
\begin{aligned}
& D \sum \sum w_{m n} \iint\left(\nabla^{4} X_{m} Y_{n}\right) X_{i} Y_{k} d x d y \\
& =\sum \sum p_{m n} \iint X_{m} Y_{n} X_{i} Y_{k} d x d y
\end{aligned}
$$

Since $X_{m}$ and $Y_{n}$ are orthogonal functions, the integrations simplify to

$$
\begin{aligned}
& D \sum \sum w_{m n} \iint_{R}\left(\nabla^{4} X_{m} Y_{n}\right) X_{m} Y_{n} d x d y \\
& =\sum \sum p_{m n} \iint_{R} X_{m}^{2} Y_{n}^{2} d x d y
\end{aligned}
$$

Hence,the solution for $W_{m n}$ could be presented in compact form as:

$$
w_{m n}=\frac{P_{m n} I m_{n}}{D I_{m n^{*}}}
$$

where $I_{m n}$ and $I_{m n^{*}}$ are symbols defined to represent the double integration problems in Equation (25). Thus:

$$
\begin{gathered}
I_{m n}=\iint_{R} X_{m}^{2} Y_{n}^{2} d x d y \\
T I_{m n^{*}} \iint_{R}\left(\nabla^{4} X_{m} Y_{n}\right) X_{m} Y_{n} d x d y
\end{gathered}
$$

\section{GALERKIN - VLASOV SOLUTION}

We define the coordinate system of the plate as shown in Fig. 1.
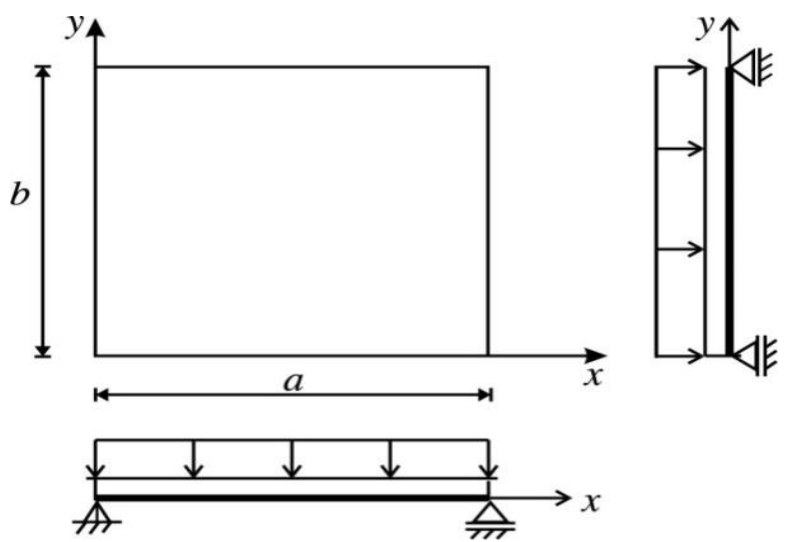

Fig. 1 Simply supported rectangular Kirchhoff plate under uniform load

The Kirchhoff plate equation is

$$
\nabla^{4} w(x, y)-\frac{p_{z}(x, y)}{D}=0
$$

In (29) $p_{z}(x, y)$ is the distributed transverse load, $D$ is the flexural rigidity of the plate $w(x, y)$ is the transverse displacement of the plate middle surface. For simply supported edges, the boundary conditions are:

$$
\begin{gathered}
w(x=0, y)=w(x=a, y)=0 \\
w(x y=0)=w(x, y=b)=0,
\end{gathered}
$$

$$
\begin{aligned}
& w_{x x}(x=0, y)=w_{x x}(x=a, y)=0 \\
& w_{y y}(x, y=0)=w(x, y=b)=0
\end{aligned}
$$

The Vlasov method uses the eigen functions of vibrating beams with identical end restraint conditions as the plate.

The equation of free vibration of uniform prismatic Bernoulli beams is

$$
\frac{\partial^{4} w(x, t)}{\partial x^{4}}=-\frac{\bar{m}}{E I} \frac{\partial^{2} w(x, t)}{\partial t^{2}}
$$

where $\bar{m}$ is the mass per unit length, $w(x, t)$ is the lateral deflection, $E$ is the Young's modulus of elasticity and $I$ is the moment of inertia.

For harmonic response

$$
w(x, t)=X(x) \sin w_{\mathrm{n}} \mathrm{t}
$$

where $w_{n}$ is the natural frequency, $X(x)$ is the shape function of the beam.

then we obtainby substitution of Equation (32) into Equation (31), we obtain:

$$
X^{i v}(x)=\frac{\bar{m} w_{n}^{2}}{E I} X(x)=\frac{\lambda^{4}}{I^{4}} X(x)
$$

where $\lambda$ is the shape parameter and $l$ is the span of the beam.

The solution of Equation (33) for simply supported ends is

$$
X_{m}(x)=\sin \frac{m \pi x}{a}, \ldots m=1,2,3, \ldots \infty
$$

Similarly, in the $y$ direction,

$$
Y_{n}(y)=\sin \frac{n \pi x}{a}, \ldots n=1,2,3, \ldots \infty
$$

Hence, by the Galerkin - Vlasov method, the displacement function is chosen as:

$$
w(x, y)=\sum_{m}^{\infty} \sum_{n}^{\infty} w_{m n} X_{m}(x) Y_{n}(y)
$$

where $X_{m}(x)$ and $Y_{n}(y)$, the basis or shape functions are the eigen functions of simply supported beams in the $x$ and $y$ coordinate directions, $w_{m n}$ are generalised coordinates.

Hence the trial displacement function is:

$$
w(x, y)=\sum_{m}^{\infty} \sum_{n}^{\infty} w_{m n} \sin \frac{m \pi x}{a} \sin \frac{n \pi x}{b} \ldots m, n
$$

$$
=1,2,3, \ldots \infty
$$

The variational form of the Kirchoff plate equation, by the Galerkin-Vlasov method becomes:

$$
\begin{aligned}
\iint_{R}\left[\nabla^{4}\left(\sum \sum w_{m n} \sin \frac{m \pi x}{a} \sin \frac{n \pi y}{b}\right)\right. \\
\left.-\frac{1}{D} \sum \sum p_{m n} \sin \frac{m \pi x}{a} \sin \frac{n \pi x}{b}\right] \\
\times \sin \frac{r \pi x}{a} \sin \frac{s \pi y}{b} d x d y=0
\end{aligned}
$$

Factoring out the $w_{m n}$ and $p_{m n}$ terms from the double integration problem in Equation (37) we obtain:

Vol. 35, No. 4, October 2016 


$$
\begin{aligned}
& \sum \sum w_{m n} \iint\left(\nabla^{4} \sin \frac{m \pi x}{a} \sin \frac{n \pi y}{b}\right) \\
& \times \sin \frac{r \pi x}{a} \sin \frac{s \pi y}{b} d x d y \\
&= \frac{1}{D} \sum \sum p_{m n} \iint\left(\sin \frac{m \pi x}{a} \sin \frac{n \pi y}{b}\right) \\
& \times\left(\sin \frac{r \pi x}{a} \sin \frac{s \pi y}{b}\right) d x d y
\end{aligned}
$$

Evaluating the biharmonic operation in the right hand side of equation (38) we obtain:

$\sum \sum w_{m n} \iint_{R}\left[\left(\frac{m \pi}{a}\right)^{4}+2\left(\frac{m \pi}{a}\right)^{2}\left(\frac{n \pi}{b}\right)^{2}\right.$

$\left.\left.+\left(\frac{n \pi}{b}\right)^{4}\right] \sin \frac{m \pi x}{a} \sin \frac{n \pi y}{b}\right) \cdot \sin \frac{r \pi x}{a} \sin \frac{s \pi y}{b} d x d y$

$=\frac{1}{D} \sum \sum p_{m n} \iint_{R} \sin \frac{m \pi x}{a} \sin \frac{n \pi y}{b} \sin \frac{r \pi x}{a} \sin \frac{s \pi y}{b} d x d y$

The vibrating simply supported beam function $\sin \frac{m \pi x}{a}, \sin \frac{n \pi y}{b}$ s are orthogonal functions. Hence for $m \neq r, n \neq s$, the integrals all vanish and are non vanishing when $m=r, n=s$. Therefore, the GalerkinVlasov variational equation simplifies to:

$$
\begin{aligned}
& \sum \sum w_{m n} \iint\left(\left(\frac{m \pi}{a}\right)^{2}\right. \\
& \left.+\left(\frac{n \pi}{b}\right)^{2}\right)^{2} \sin ^{2} \frac{m \pi x}{a} \sin ^{2} \frac{n \pi y}{b} d x d y \\
& =\frac{1}{D} \sum \sum p_{m n} \iint \sin ^{2} \frac{m \pi x}{a} \sin ^{2} \frac{n \pi y}{b} d x d y \\
& =\iint_{R} \sin ^{2} \frac{m \pi x}{a} \sin ^{2} \frac{n \pi y}{b} d x d y
\end{aligned}
$$

Then

$$
\begin{array}{r}
\sum \sum w_{m n}\left[\left(\frac{m \pi}{a}\right)^{2}+\left(\frac{n \pi}{a}\right)^{2}\right]^{2} I_{m n} \\
=\frac{1}{D} \sum \sum P_{m n} I_{m n}
\end{array}
$$

For any $m, n$,

$$
w_{m n}=\frac{p_{m n}}{D\left(\left(\frac{m \pi}{a}\right)^{2}+\left(\frac{n \pi}{b}\right)^{2}\right)^{2}}=\frac{p_{m n}}{\pi^{4} D\left(\frac{m^{2}}{a^{2}}+\frac{n^{2}}{b^{2}}\right)^{2}}
$$

$p_{m n}$ are the Fourier series coefficients of the load function. For uniformly distributed loads of intensity $p_{0}$,

$$
\begin{aligned}
p_{m n}=\frac{4}{a b} \int_{0}^{a} \int_{0}^{b} p_{0} & \sin \frac{m \pi x}{a} \sin \frac{n \pi y}{b} d x d y \\
& =\frac{4 p_{0}}{a b} \int_{0}^{b} \sin \frac{n \pi y}{b} d y=\frac{16 p_{0}}{m n \pi^{2}}
\end{aligned}
$$

$m, n=1,3,5,7, \ldots$

Hence for uniformly distributed loads,

$$
\begin{aligned}
& w(x, y) \\
& =\sum \sum w_{m n} \sin \frac{m \pi x}{a} \sin \frac{n \pi y}{b} \\
& =\frac{16 p_{0}}{D \pi^{6}} \sum_{m}^{\infty} \sum_{n}^{\infty} \frac{\sin \frac{m \pi x}{a} \sin \frac{n \pi y}{b}}{\left(\frac{m^{2}}{a^{2}}+\frac{n^{2}}{b^{2}}\right)^{2}}
\end{aligned}
$$

$m, n=1,3,5,7, \ldots$

\subsection{Maximum Deflection}

The maximum deflection occurs at the plate center, and is obtained as:

$$
\begin{aligned}
& w_{\max }=w\left(x=\frac{a}{2}, y=\frac{b}{2}\right) \\
& =\frac{16 p_{0}}{D \pi^{6}} \sum_{m}^{\infty} \sum_{n}^{\infty} \frac{\sin \frac{m \pi x}{2} \sin \frac{n \pi y}{2}}{\left(\frac{m^{2}}{a^{2}}+\frac{n^{2}}{b^{2}}\right)^{2}} \\
& \frac{16 p_{0}}{D \pi^{6}} \sum \sum \frac{-1\left(\frac{m+n-2}{2}\right)}{m n\left(\frac{m^{2}}{a^{2}}+\frac{n^{2}}{b^{2}}\right)^{2}}
\end{aligned}
$$

\subsection{Bending Moment Distributions}

The bending moment distributions are found from the moment displacement relations:

$$
\begin{aligned}
& M_{x x}=-D\left(w_{x x}+\mu w_{y y}\right) \\
& M_{y y}=-D\left(w_{y y}+\mu w_{x x}\right)
\end{aligned}
$$

Thus,

$$
\begin{aligned}
& M_{x x}=\frac{16 p_{0}}{D \pi^{4}} \sum_{m}^{\infty} \sum_{n}^{\infty} \frac{\left(\frac{m^{2}}{a^{2}}+\mu \frac{n^{2}}{b^{2}}\right) \sin \frac{m \pi x}{a} \sin \frac{n \pi y}{b}}{m n\left(\frac{m^{2}}{a^{2}}+\frac{n^{2}}{b^{2}}\right)^{2}} \\
& M_{y y}=\frac{16 p_{0}}{D \pi^{4}} \sum_{m}^{\infty} \sum_{n}^{\infty} \frac{\left(\frac{n^{2}}{b^{2}}+\mu \frac{m^{2}}{a^{2}}\right) \sin \frac{m \pi x}{a} \sin \frac{n \pi y}{b}}{m n\left(\frac{m^{2}}{a^{2}}+\frac{n^{2}}{b^{2}}\right)^{2}}
\end{aligned}
$$

At the center of the plate, the bending moments are

$$
\begin{aligned}
& M_{x x}=\frac{16 p_{0}}{D \pi^{4}} \sum_{m}^{\infty} \sum_{n}^{\infty} \frac{\left(\frac{m^{2}}{a^{2}}+\mu \frac{n^{2}}{b^{2}}\right)(-1)^{\left(\frac{m+n-2}{2}\right)^{2}}}{m n\left(\frac{m^{2}}{a^{2}}+\frac{n^{2}}{b^{2}}\right)^{2}} \\
& M_{y y}=\frac{16 p_{0}}{D \pi^{4}} \sum_{m}^{\infty} \sum_{n}^{\infty} \frac{\left(\frac{n^{2}}{a^{2}}+\mu \frac{m^{2}}{b^{2}}\right)(-1)^{\left(\frac{m+n-2}{2}\right)^{2}}}{m n\left(\frac{m^{2}}{a^{2}}+\frac{n^{2}}{b^{2}}\right)^{2}}
\end{aligned}
$$

The Galerkin-Vlasov result for $W_{\max }, M_{x x}$ and $M_{y y}$ obtained at the center of uniformly loaded Kirchhoff plate for various aspect ratios of the plate are tabulated in Table 1.

\section{DISCUSSION OF RESULTS AND CONCLUSION}

In this work, the Galerkin-Vlasov variational method has been used to solve the problem of bending of 
rectangular Kirchhoff plate with simply supported edges and under uniformly distributed load over the entire plate region. Orthogonal basis functions which were the beam vibration modal shape functions were used to describe the displacement shape functions of the plate, yielding considerable simplifications in the evaluation of the integration problem.

Table 1:Galerkin-Vlasov solutions for maximum deflection and maximum bending moments in SSSS Kirchhoff Plates under uniform load on the entire plate

\begin{tabular}{ccccc}
\multicolumn{5}{c}{ region $0 \leq x \leq a, 0 \leq y \leq b ; w_{\max }=\delta_{1} \frac{p_{0} a^{4}}{E t^{3}}, \rightarrow$} \\
\multicolumn{5}{c}{$M_{\text {xxmax }}=\delta_{2} p_{0} a^{2},=w_{\text {max }}=\delta_{4} \frac{p_{0} a^{4}}{D}, M_{\text {yymax }}=\delta_{3} p_{0} a^{2}$} \\
\hline$b / a$ & $\delta_{1}$ & $\delta_{2}$ & $\delta_{3}$ & $\delta_{4}$ \\
\hline 1 & 0.0443 & 0.0477 & 0.0479 & $4.062 \times 10^{-3}$ \\
1.2 & 0.0616 & 0.0626 & 0.0501 & $5.641 \times 10^{-3}$ \\
1.4 & 0.0770 & 0.0753 & 0.0506 & $7.07 \times 10^{-3}$ \\
1.5 & & 0.0812 & & $7.724 \times 10^{-3}$ \\
1.6 & 0.0906 & 0.0862 & 0.0493 & $8.293 \times 10^{-3}$ \\
1.8 & 0.1017 & 0.0948 & 0.0479 & $9.313 \times 10^{3}$ \\
2 & 0.1106 & 0.1017 & 0.0404 & $10.129 \times 10^{-3}$ \\
3 & 0.1336 & 0.1189 & 0.0404 & $12.233 \times 10^{-3}$ \\
4 & 0.1400 & 0.1235 & 0.0384 & $12.819 \times 10^{-3}$ \\
5 & & 0.1246 & 0.03775 & $12.971 \times 10^{-3}$ \\
$\infty$ & 0.1422 & 0.1250 & 0.0375 & $13.021 \times 10^{-3}$ \\
\hline
\end{tabular}

For simply supported edges, the general Galerkin Vlasov solution is presented as Equantion (26). The displacement function for a simply supported rectangular Kirchhoff plate was found in this work to be exactly identical with the Navier solution, and is presented in Equation (45).

The displacement function is found to be a rapidly convergent series of infinite terms and a few terms of the series gives satisfactory results for the maximum deflection which was found to occur at the plate center.Similarly, the bending moment functions were obtained by using the moment - displacement equations, and the maximum bending moments were found to occur at the centre of the plate. The bending moment distributions and the maximum values about the $x$ and $y$ coordinate axes were found to be given by Equations (50) - (53).

The deflections and bending moments were evaluated at different aspect ratios of the plate and the variation presented in Table 1. The Galerkin-Vlasov solutions for the variation of deflections and bending moments in simply supported rectangular Kirchhoff plates under uniformly distributed loads are exactly identical with the solutions obtained using the Navier's double trigonometric series method. The Galerkin-Vlasov solutions for maximum deflection and maximum bending moment for various plate aspect ratios as presented in Table 1 agree with classical solutions in the technical literature.

\section{REFERENCES}

[1] Ventsel, E. and Krauthammer, T. Thin Plates and Shells Marcel Dekker Inc. USA pp. 1 - 90. 2001.

[2] Mansfield, E. H. The bending and stretching of plates. Cambridge University Press, Cambridge pp. $1-25,1989$.

[3] Timoshenko, S. P. and Woinowsky - Krieger, Theory of Plates and Shells McGraw Hill Book Co. London pp. 105 - 179. 1959.

[4] Vinson, Jack R. Solid Mechanics and its applications - plate and panel structures of isotropic sandwich construction.Springer, Dordrecht, the Netherlands pp. $43-44.2005$.

[5] Szilard, R. Theories and Applications of Plate Analysis Classical Numerical and Engineering Methods. John Wiley and Son Inc. New Jersey, pp45 - 46. 2004.

[6] Chen, P. S. and Archer, N. N Solutions of a Twelfth Order Thick Plate Theory. Acta Mechanics, 79, pp77-111 Springer, Verlag. 1989.

[7] Mindlin, R. D. Influence of Rotary Inertia and Shear on Flexural Motions of Isotropic Elastic Plates. J. Applied Mechanics. Vol. 18 No. 1. Pp 31 - 38. 1951.

[8] Bletzinger, K. Theory of Plates Part II Plates in Bending Lecture Notes Winter Semester 2012/2013. Lehrstuhl für Statik Technische Univasitat Munchen October 2008. http//www.saribd.com/doc/112345132/Theory of plates-part 2. 2008.

[9] Durban, D., Givoli, D. and Simnonds, J.G. (Eds.) Solid Mechanics and its Applications Advances in the Mechanics of Plates and Shells: The Avio nvan Libai Anniversary Volume, Kluwer Academic Publishers, Dordrecht, The Netherlands. 2001. 